\title{
Historias del bienestar. Desde la historia de las emociones a las políticas de la experiencia
}

\author{
Javier Moscoso SANABria \\ Centro de Ciencias Humanas y Sociales / CSIC \\ Javier.moscoso@cchs.csic.es \\ Juan Manuel Zaragoza BernaL \\ Centre for the History of the Emotions / Queen Mary University of London \\ j.m.zaragoza@qmul.ac.uk
}

Recibido: 26/06/2014

Aceptado: 02/07/2014

\section{RESUMEN}

El propósito de este texto es doble. Por un lado, haremos un pequeño recorrido, inevitablemente incompleto, por lo que ha sido la historia de las emociones en España y Latinoamérica. En segundo lugar, argumentaremos a favor de una orientación metodológica centrada en la historia de las experiencias subjetivas y, más en particular en este caso, en la historia cultural del bienestar. Defendemos, por último, que el proyecto de cooperación entre nuestro grupo de Madrid y un equipo de médicos, psicólogos y psiquiatras de Valencia en torno a la enfermedad de la fibromialgia, constituye un buen ejemplo de relación cooperativa entre las humanidades y las ciencias de la salud en el siglo XXI.

Palabras clave: Emociones, Experiencia, América Latina, Bienestar, Fibromialgia.

\section{Histories of Wellbeing. From the History of the Emotions to the Politics of the Experience}

\begin{abstract}
The aim of this paper is twofold. On the one hand, we intend to produce a short review on the history of the emotions in Spain and Latin America. On the other hand, we will argue in favor of a methodological approach, focused on the history of subjective experiences and, specifically in this case, on the cultural history of wellbeing. We will conclude that the cooperative project developed by our group of Madrid and a Valencian team of doctors, psychologists and psychiatrists specialized in the study and treatment of fibromyalgia is a good example of how cooperative endeavor between the Humanities and the Health Sciences in the XXI century.
\end{abstract}

Key words: Emotions, Experience, South America, Wellbeing, Fibromyalgia. 
Sumario: Introducción. 1. La historia de las emociones y la política de la experiencia. 2. Experiencia y Relato. 2.1. Historia cultural del bienestar. 2.2. El caso de la fibromialgia 3. Conclusiones. 4. Referencias bibliográficas.

\section{Introducción $^{1}$}

La historia de las emociones, que se ha desarrollado como disciplina académica en los últimos años, conlleva una toma de postura en relación a dos grandes problemas. En primer lugar, los proponentes de este enfoque buscan antecedentes históricos, de modo que su tarea se vea legitimada por algunas grandes figuras del pasado. En muchos textos pervive el deseo de encontrar un origen común, una historia canónica de esta nueva corriente historiográfica ${ }^{2}$. En segundo lugar, no son pocos quienes intentan arrojar luz sobre algunos de los problemas metodológicos o teóricos a los que estos estudios históricos han dado lugar ${ }^{3}$. La respuesta a ambas dificultades viene determinada por contextos nacionales y variaciones que podríamos considerar "regionales", tanto en el sentido de que dependen de su localización geográfica como del punto de partida. En el caso de los Cultural Studies que se practican en USA, y que parten en su mayoría de Departamentos de Análisis del Discurso y de Literatura Comparada, el carácter transversal se percibe sobre todo en un interés por la antropología, por la cultura visual y material. Los estudios de historia de las emociones del Reino Unido y de Alemania contrastan a su vez con lo que los historiadores franceses, y muchos latinoamericanos, prefieren llamar "historia de las sensibilidades". Tampoco sus problemas son los mismos. Una comparación de los textos programáticos de Peter Burke, Jo Labanyi, Peter Stearns, Ute Frevert y Barbara Rossenwein, por mencionar a algunos de los autores de mayor impacto, arroja resultados diferentes dependiendo de la orientación metodológica inicial ${ }^{4}$. Esto no quiere decir que no se reconozcan

1 La realización de este texto ha sido posible gracias a la financiación recibida en dos proyectos de investigación otorgados por el Ministerio de Economía y Competitividad del Gobierno de España: FFI2010-20876: Epistemología Histórica: Historia de las emociones en los siglos XIX y XX) y FFI2013-46361: Subjective and Objective Elements in the Cultural History of Wellbeing: 1750-1950).

2 Se suele citar a Lucien Febvre, y su texto de 1940, como el punto de arranque de esta nueva disciplina, ver FEBVRE, Lucien: "La sensibilité et l'histoire. Comment reconstituer la vie affective d'autrefois?," Annales d'Histoire sociale, 2 (1941), pp. 5-20. Para una revisión de la historia de las emociones desde la década de 1940, ver ROSENWEIN, Barbara H., "Worryng about Emotions in History," The American Historical Review, 107 (2002), pp. 821-845. En este artículo, Rosenwein proponía las "comunidades emocionales" o "los sistemas afectivos" como herramientas heurísticas, idea que desarrollaría en otra de su obra, ROSENWEIN, Barbara H.: Emotional Communities in the Early Middle Ages, Ithaca \& London, Cornell University Press, 2006.

3 MOSCOSO, Javier, "Poétique, rhétorique et politique des émotions: le drame de l'expérience," en LANGUE, Frédérique y CAPDEVILLE, Luc (eds.): Le passé des émotions. D'une Histoire à vif en Espagne et Amérique Latine, Rennes, Presses Universitaires de Rennes (en prensa).

4 Véase los recientes libros de FREVERT, Ute et al.: Emotional Lexicons: Continuity and Change in the Vocabulary of Feeling 1700-2000, Oxford, Oxford University Press, 2014. Especialmente capítulo 1, Frevert: "Defining Emotions: Concepts and Debates over Three Centuries"; y MATT, Susan J. y STEARNS, Peter N.: Doing Emotions History, Urbana, University of Illinois Press, 2013. En concreto la introducción, firmada por ambos, y el excelente texto de Stearns, que abre el libro, "Modern Patterns in Emotions History", pp. 17-40. Véase también ROSENWEIN, Barbara H.: "Problems and Methods in the History of Emotions," Passions in Context, 1 (2010), pp. 1-32; BURKE, Peter: "Is There a Cultural History of the Emotions?," en GOUK, 
las dificultades que, desde otras ópticas, se plantean en los estudios de historia de las emociones; todo lo contrario: este reconocimiento no evita una predilección a la hora de adaptarse a las demandas locales.

Estas preferencias también se aplican a nuestro grupo de investigación en Madrid, que ha optado por la denominación "historia de la experiencia" frente a otras más asentadas. En otro lugar, hemos querido sugerir cómo este enfoque se centra en el estudio de las formas culturales de la subjetividad, incluyendo las emociones, por supuesto, pero también las sensaciones, las pasiones, o los instintos. De modo más general, nuestra historia de la experiencia no está interesada en la referencialidad de los estados de la conciencia, sino en las condiciones que hacen posible su carácter culturalmente significativo. La referencialidad de los estados emocionales, aquello a lo que las emociones inicialmente se refieren o lo que en apariencia significan, nos interesa menos que las formas culturales que las hacen posibles. Es justamente el carácter dramático de la experiencia emocional lo que permite asentar su estudio sobre sus elementos poéticos, retóricos y políticos. Nuestra aproximación, más preocupada por la filosofía de la historia que por la del lenguaje, permite ampliar el espectro de nuestro trabajo, tanto en el sentido de promover investigaciones relacionadas con la historia de experiencias que son sin duda emocionales, aunque no solo emocionales (como la historia de la enfermedad, del cuidado, de la infancia, o de la experiencia alterada), cuanto por lo que respecta a los intentos de generar sinergias con grupos de investigación que, provenientes de las ciencias biomédicas, comparten algunos de nuestros presupuestos metodológicos.

El propósito de este texto es doble. Por un lado, haremos un pequeño recorrido, inevitablemente incompleto, por lo que ha sido la historia de las emociones en España, incluyendo también algunas referencias puntuales sobre los contextos latinoamericanos. Esta somera presentación de algunos de los hitos en el campo de la historia de las emociones escrita en español sugiere una nueva manera de abordar la historia de las experiencias subjetivas en un contexto post-colonial, atendiendo a singularidades propias de una región inmensa del planeta. No se trata únicamente de acceder a un mercado de más de quinientos millones de personas, sino de ofrecer una perspectiva "desde el Sur", capaz de poner el acento en las especificidades locales antes que en los constructos teóricos heredados ${ }^{5}$. Nuestra propuesta se atiene a lo que podría denominarse "política de las emociones": la reflexión crítica sobre el papel del propio observador a la hora de configurar el objeto de estudio; una perspectiva que

Penelope y HILLS, Helen (eds.): Representing Emotions: New Connections in the Histories of Art, Music and Medicine, Aldershot, Ashgate, 2005, pp. 35-48; LABANYI, Jo: "Doing Things: Emotions, Affect, and Materiality," Journal of Spanish Cultural Studies 11 (2010), pp. 223-33. Otros autores que realizan propuestas similares son SCHEER, Monique: "Are Emotions a Kind of Practice (and Is That What Makes Them Have a History)? A Bourdieuan Approach to Defining Emotion", History and Theory, 51 (2012), pp. 193-220. También, GOLDIE, Peter: The Mess inside: Narrative, Emotion, and the Mind, Oxford, Oxford University Press, 2012, pp. 57-75.

5 La creación en abril de 2014 de la lista de distribución sobre Historia de la Experiencia Emocional (H-EXEM), en REDIRIS, busca convertirse en un foro de discusión entre la comunidad de historiadores de las emociones en español a ambos lados del Atlántico, así como favorecer el intercambio de opiniones y la generación de nuevas iniciativas de colaboración. 
no debe confundirse con la historia de las emociones políticas, o con otras corrientes filosóficas relacionadas con la regulación pública de las pasiones ${ }^{6}$.

En segundo lugar, pondremos un ejemplo de la relación entre esta aproximación metodológica en el contexto de las humanidades médicas. El caso a exponer es un proyecto de cooperación entre nuestro grupo de Madrid y un equipo de médicos, psicólogos y psiquiatras de Valencia. No es el único posible, pero pensamos que constituye un buen ejemplo de cómo se puede abordar tanto el problema de la relación cooperativa de las ciencias cuanto la confluencia de planteamientos metodológicos comunes en torno al pasado y al presente de esas coloraciones del flujo de la vida que denominamos "emociones" . La investigación sobre la experiencia del paciente de fibromialgia, que es la que vamos examinar, plantea varios retos: incrementar la cooperación con personal clínico, pero también con pacientes y usuarios potenciales; definir un marco de actuación de las Humanidades Médicas como un espacio de interacción entre las disciplinas cuantitativas y las cualitativas que pueda producir un encuentro entre las ciencias, las humanidades y las artes; presentar modelos culturalmente significativos y socialmente relevantes de la relación entre la salud y el bienestar; y, por último, fomentar el uso de nuevas tecnologías tanto en el ámbito de la investigación, como en la transferencia y divulgación de resultados.

\section{La historia de las emociones y la política de la experiencia}

Como en otros lugares del mundo, la historia de las emociones escrita en español, o publicada por miembros de comunidades científicas iberoamericanas, también ha recibido un impulso importante, muchas veces a pesar de la indiferencia de las instituciones, cuando no de su abierto rechazo. Si fuera verdad, como sugiere Reinhart Koselleck, que las revoluciones historiográficas siempre surgen de los derrotados, habría que preguntarse qué pasiones o emociones del pasado quedaron silenciadas por la historiografía de la victoria ${ }^{8}$. En la Península Ibérica, muchos historiadores han sido partícipes y protagonistas de esta nueva corriente desde sus mismos inicios. Es el caso, por poner un ejemplo, de la investigadora de la Universidad Complutense Lucía Díaz Marroquín, que ya en 2004 publicaba en Revista de Literatura un artículo titulado "La nieve arder. La retórica afectiva en el universo petrarquista de la zarzuela Acis $y$ Galatea" . Esta joven estudiosa de la retórica musical también daría a la imprenta, en 2008, la que tal vez sea la primera monografía en español que puede encuadrarse dentro de la historia de las emociones strictu sensu. En su La retórica de los afectos, Díaz Marroquín describe cómo la retórica, tanto clásica como de raíz cristiana, refle-

\footnotetext{
6 Sobre este asunto, véase por ejemplo NUSSBAUM, Martha C.: Political Emotions: Why Love Matters for Justice, Cambridge, The Belknap Press of Harvard University Press, 2013.

7 Sobre las emociones como intensificaciones de la experiencia, véase DEWEY, John: Art as Experience, London, George Allen \& Unwin, 1934.

8 KOSELLECK, Reinhart: "Cambio de experiencia y cambio de método," en Los estratos del tiempo: estudios sobre la historia, Barcelona, Ediciones Paidós, 2001, pp. 43-93.

9 DÍAZ MARROQUÍN, Lucía: “'La nieve arder'. La retórica afectiva en el universo petrarquista de la zarzuela Acis y Galatea," Revista de Literatura, 66, 132 (2004), pp. 431-464.
} 
jaba la tensión existente entre los dos polos entre los que se sitúa, tradicionalmente, el equilibrio psíquico: la ética racional y lo patético o pasional ${ }^{10}$. Su estudio no sólo llamó la atención por lo temprano de su aparición, sino por la variedad de sus fuentes, que incluían testimonios literarios, musicales y visuales.

También en fecha muy temprana encontramos el primer curso universitario dedicado a la historia de las emociones organizado por María Tausiet y James S. Amelang con el título Accidentes del alma. Las emociones en la Edad Moderna, que se celebró en junio de 2006 en el marco de los Cursos de Verano de la Universidad Complutense en El Escorial, y que sería el germen del que surgiría el libro del mismo título publicado por Abada en 2009. Éste recogía algunas de las presentaciones de dicho curso, junto a otros artículos de nuevo cuño, organizados alrededor de tres ejes: códigos emocionales, la emoción ritualizada, y la expresión de las emociones. Se trataba de un libro desigual donde excelentes textos, como el dedicado al llanto ritual firmado por W.A. Christian Jr., conviven con otros menos reseñables ${ }^{11}$. Más allá de esta fragilidad propia de casi cualquier libro colectivo, el de Tausiet y Amelang comparte las fortalezas, pero también las debilidades, de otras monografías producidas en el contexto internacional: la filiación con la nueva historia cultural, la indefinición del concepto de emoción, o la excelente relectura de fuentes que ayudan a iluminar nuevos aspectos de la vida emocional del pasado ${ }^{12}$.

También por esos años, más concretamente en 2007, se fundaba el grupo de investigación "History of Experience" (HIST-EX) en el Centro de Ciencias Humanas y Sociales (CCHS) de Madrid. Este grupo, junto con el dirigido por la profesora Pura Fernández, también del CCHS, emprendió proyectos de colaboración con diversas instituciones e investigadores internacionales. Así, el grupo de Pura Fernández organizó, junto a Jo Labanyi de la New York University y Luisa-Elena Delgado, de la University of Illinois at Urbana-Champaign, el congreso internacional "Conocer, conmover", que contaría con dos ediciones, una celebrada en marzo de 2010 en Madrid y la otra en Nueva York, en abril de $2011^{13}$. Por su parte, el grupo HIST-EX comenzó a colaborar con el grupo entonces liderado por Bernardino Fantini en la Universidad de Ginebra; producto de esta colaboración internacional fue el congreso, celebrado en octubre de 2011, titulado "On Resentment: an International Workshop on the History of the Emotions" ${ }^{14}$. El esfuerzo de ambos proyectos se concretó en la realización en Madrid de dos workshops en 2010 y 2011, con el título "Cultural History of the Emotions: International Meeting of Young Researchers", que contaron con la presencia de estudiantes y doctorandos provenientes de un buen número de instituciones europeas.

10 DÍAZ MARROQUÍN, Lucía: La retórica de los afectos (Edition Reichenberger, 2008).

11 TAUSIET, Carles María y AMELANG, James S. (eds.): Accidentes del alma: las emociones en la Edad Moderna, Madrid, Abada, 2009.

12 ZARAGOZA BERNAL, Juan Manuel: "Historia de las emociones: una corriente historiográfica en expansión,” Asclepio, 65, 1 (2013): e012, doi:10.3989/asclepio.2013.12.

13 Publicado por SIECE, “GRAFOSFERA: conocer, conmover". Disponible en:

http:/grafosfera.blogspot.co.uk/2010/03/conocer-conmover.html [consultado el 30 de mayo de 2014].

14 FANTINI, Bernardino, MARTÍN MORUNO, Dolores y MOSCOSO, Javier: On Resentment: Past and Present, Cambridge, Cambridge Scholars, 2013. 
Más allá de lo logrado por estos grupos de investigación, la historia de las emociones en España cuenta con numerosos practicantes en distintas universidades y centros de investigación. Es el caso del profesor de Filología Moderna de la Universidad de Castilla-La Mancha, Javier E. Díaz Vera, cuyos estudios sobre el vocabulario emocional del inglés medieval y renacentista, con especial énfasis en el estudio de la metáfora como productora de significados, han sido publicados en relevantes revistas internacionales ${ }^{15}$. O el reciente libro de Rosa Medina Doménech, profesora de Historia de la Medicina de la Universidad de Granada: una historia del amor en los primeros años del franquismo y sus vínculos con la ciencia; un texto, este último, que puede encuadrarse en la relación, cada vez más fructífera, que la historia de las emociones mantiene, prácticamente desde sus inicios, con los estudios de género pero también con la historia de la medicina y de la ciencia ${ }^{16}$. Al mismo tiempo, proliferan en España los equipos de investigación, que provenientes de la literatura y de los estudios de cultura visual y material profundizan en aspectos relacionados con el pasado y el presente de las emociones. Algunos de ellos, como el liderado por Helena González, se encuentran en Barcelona, ligados al Institut d'Humanitats.

Los estudios emocionales en Latinoamérica no sólo gozan de una larga historia, sino que ofrecen una perspectiva propia que sitúa las emociones dentro del discurso político, muchas veces reivindicativo. Podemos situar su eclosión en el año 2002, cuando el Grupo de Pesquisa em Antropologia e Sociologia das Emoçoes, fundado en 1994, publica el primer número de la Revista Brasileira de Sociologia da Emoçao. En la editorial del número inaugural, firmada por Mauro Guilherme Pinheiro Koury, el grupo menciona a autores clásicos como Durkheim, Simmel, y Weber, pero también a Elias y Giddens, como sus referentes intelectuales. ${ }^{17} \mathrm{La}$ importancia de esta revista no radica únicamente en su carácter pionero, sino en que presentan los estudios emocionales en una perspectiva política y social propia del contexto latinoamericano. Siete años más tarde, en 2009, aparece la Revista Latinoamericana de Estudios sobre Cuerpos, Emociones y Sociedad, publicada en Argentina e impulsada por la Red Latinoamericana de Estudios Sociales sobre las Emociones y los Cuerpos. Empleando conceptos como el de "economía política de las sensaciones", y situando el cuerpo y las emociones en el eje vertebrador de los procesos de estructuración social, esta revista, y la Red de la que surge, también insisten en la pertinencia de situar las emociones en una perspectiva política. ${ }^{18} \mathrm{El}$ énfasis entre la relación cuer-

15 DÍAZ-VERA, Javier y CABALLERO, Rosario: "Exploring the Feeling-Emotions Continuum across Cultures: Jealousy in English and Spanish," Intercultural Pragmatics, 10, no. 2 (2013), pp. 265-94, doi:10.1515/ip-2013-0012; DÍAZ-VERA, Javier: “On Saying Two Things at Once: The Historical Semantics and Pragmatics of Old English Emotion Words," Folia Linguistica Historica, vol. 35, no. 1 (2014); DÍAZVERA, Javier: "Emotions in the Household: Emotion Words and Metaphors in Domesday Book Personal Names," Names - A Journal of Onomastics, 2015.

16 MEDINA DOMÉNECH, Rosa María: Ciencia y sabiduría del amor: una historia cultural del franquismo (1940-1960), Madrid- Frankfurt am Main, Iberoamericana, 2013.

17 PINHEIRO KOURY, Mauro Guilherme: "Surge uma revista em sociologia das emoções," Revista Brasileira de Sociologia da Emoçao 1, no. 1 (2002), pp. 4-5; BENDELOW, Gillian y WILLIAMS, Simon J.: Emotions in Social Life: Critical Themes and Contemporary Issues, Abingdon (Oxon)-Nueva York, Routledge, 2002.

18 Cuerpos, Emociones y Sociedad, “Presentación,” Revista Latinoamericana de Estudios Sobre Cuerpos, Emociones Y Sociedad 1, no. 1 (2009), pp. 4-5. 
po y afectividad será otra de las características sobresalientes de la aproximación latinoamericana a los estudios emocionales. ${ }^{19}$ Una perspectiva que entronca con los estudios sobre biopolítica, tan exitosos en el continente, y que varios autores sitúan en una segunda, cuando no tercera, generación. Esto es lo que argumenta, por ejemplo, el libro coordinado por Ivan Pincheira, profesor del Departamento de Sociología de la Universidad de Chile, bajo el título Archivos de Frontera. El gobierno de las emociones en Argentina y Chile del presente, que relaciona biopolítica y emociones para concentrar su análisis en "los nexos existentes entre el plano emotivo y las contemporáneas prácticas gubernamentales". ${ }^{20}$

Otros trabajos que se vienen realizando en los últimos años trasladan este enfoque político de las emociones a la investigación en humanidades en un sentido amplio. El proyecto dirigido por Jane-Dale Lloyd, de la Universidad Iberoamericana, de México D.F sobre la historia del tiempo presente busca historiar el pasado reciente de la sociedad mexicana, a través de la "experiencia propia" sin evitar la problemática moral y política que tal indagación del pasado "vivo" puede y debe sacar a la luz. La profesora Lloyd lidera un grupo multidisciplinar y transversal, lo que se refleja en los distintos proyectos de investigación individuales que convergen en el general, y que van desde las relaciones Iglesia-Estado en el México de los años cincuenta hasta una aproximación fenomenológica a las relaciones entre memoria, experiencia e historia. Otro proyecto de investigación que merece la pena señalar es el liderado por Johanna Lozoya, en la UNAM. El Laboratorio Grupo Estudio de las Emociones: Ciudad \& Emociones es, al igual que el anterior, un grupo multidisciplinar, centrado esta vez en el estudio de los espacios urbanos como generadores de comunidades emocionales que a su vez permitan una mayor cohesión social. El énfasis en los estudios sobre las configuraciones del espacio urbano, así como en la relación entre esos la cultura material y las formas colectivas de experiencia configura un proyecto de investigación único, en el que confluyen perspectivas metodológicas muy variadas.

La política de las emociones no se restringe únicamente a enfoques sociológicos, sino que se extiende también a otros estudios provenientes de las humanidades. En todos ellos lo que pervive es la relación esquiva entre el presente y el pasado, que coloca al historiador en la necesidad de ubicar su investigación en el contexto local, antes que en el incierto espacio de las verdades eternas. Sin menoscabo de otras investigaciones mucho más teóricas, el rasgo definitorio de los estudios sobre teoría e historia de las emociones que se vienen desarrollando en el contexto Iberoamericano sugiere una mayor preocupación por la posición del observador, así como por las circunstancias específicas que posibilitan la relevancia social de la investigación en curso. En este sentido, la historia de las emociones surge de un compromiso relativo a la necesidad de hacer historia desde y para el presente, no sólo por las limitaciones presupuestarias que, en muchas ocasiones, obligan a definir campos de actuación específicos, sino por la propia delimitación y naturaleza de las emociones del presente y del pasado en el contexto Latino americano.

19 SABIDO RAMOS, Olga: "El cuerpo y la afectividad como objetos de estudio en América Latina: intereses temáticos y proceso de institucionalización reciente," Sociológica (México) 26, no. 74 (2011), pp. 33-78.

20 PINCHEIRA, Iván (ed.), Archivos de frontera. El Gobierno de las emociones en Argentina y Chile del presente, Santiago de Chile, Ediciones Escaparate, 2012, p. 28. 
La forma que adopta esa relación entre lo de antes y lo de ahora también se encuentra presente en nuestro grupo de investigación en Madrid, y especialmente en uno de nuestros últimos proyectos. La historia cultural del bienestar y, de modo más particular, el estudio y tratamiento de la enfermedad de fibromialgia participan de esa tensión intelectual entre la demanda social y las exigencias académicas. El propósito no puede ser tan sólo desentrañar acríticamente las emociones del pasado, sino hacer visible un relato olvidado que, a su vez, permita una mejor comprensión de nuestro mundo. En el contexto latinoamericano, la historia de las emociones vertebra una nueva forma de organización académica que mientras sugiere una actividad cooperativa entre disciplinas académicas variadas también defiende la relevancia social de las nuevas humanidades en el contexto de la sociedad global del siglo XXI.

\section{Experiencia y relato}

La relación entre la percepción subjetiva y la consideración objetiva de la salud constituye uno de los temas de mayor alcance en el contexto de la cultura económica del mundo contemporáneo. La definición de la UNESCO, que entiende la salud como un estado de bienestar físico, psicológico y social, ha sido criticada por su generalidad conceptual, pero no por su incapacidad a la hora de calibrar el significado de una experiencia cambiante. Al contrario que en el planteamiento universalista del libro de Bergdolt, Wellbeing. A Cultural History, que considera invariables los elementos de la salud, una historia cultural del bienestar debe esclarecer las variaciones históricas en la percepción subjetiva de la experiencia y, más específicamente, debe arrojar luz sobre las condiciones que permiten la valoración positiva de esa misma experiencia ${ }^{21}$. Este tipo de investigación tiene interés en dos ámbitos diferenciados. En primer lugar, atañe a la historia de la medicina y, de modo más general, confiere relevancia y centralidad a la historia de los estados subjetivos de conciencia. En segundo lugar, también permite una cooperación fructífera entre las humanidades y las ciencias biomédicas. Ambos aspectos merecen tratamiento diferenciado.

\subsection{Historia cultural del bienestar}

Es bien sabido que la Ilustración vio nacer y desarrollarse una concepción secularizada de la salud, ligada a una ética de la responsabilidad y a una política de educación pública. La medicalización de la salud y su consideración como asunto de Estado tuvieron efectos muy variados en las políticas públicas: desde el nacimiento de la dietética o de la gimnasia escolar hasta la supervisión de la nutrición o de los usos del agua. ${ }^{22} \mathrm{~A}$ estos ejemplos podrían añadirse otros muchos, como el control de la natalidad, la seguridad laboral, el cuidado de la pobreza, así como la regulación de

21 BERGOLD, Klaus: Wellbeing: A Cultural History of Healthy Living, Cambridge-Malden (MA), Polity, 2008.

22 GOUBERT, Jean-Pierre: Une histoire de l'hygiène: eau et salubrité dans la France contemporaine, vol. 1, Paris, Pluriel, 2011. 
la sexualidad o del parto $^{23}$. La subordinación de la vida a principios de naturaleza científica continuó durante todo el siglo XIX con el desarrollo de la bacteriología y de la higiene social, y desembocó tanto en los planteamientos eugenésicos de principios del siglo XX, como en el estado del bienestar posterior a la Segunda Guerra Mundial ${ }^{24}$. El nacimiento de la medicina clínica, la formación de nuevos cuadros profesionales, así como la creación de centros hospitalarios y de investigación formaron parte de esa política de regulación del bienestar social. Tanto el nuevo humanitarismo, por un lado, como la puesta en valor de un sistema de higiene pública, por el otro, promovieron la reforma de hospitales, prisiones, cementerios, pero también la promulgación de leyes relacionadas con la limitación del trabajo infantil o con las medidas de re-urbanización y saneamiento de lugares insalubres. ${ }^{25}$ Durante el siglo XIX, al mismo tiempo que se incentivaban campañas de vacunación o de profilaxis ligadas, por ejemplo, a la lucha contra la viruela, la sífilis o el cólera, se promulgaron leyes relacionadas con la educación social, con el maltrato infantil o con el mundo laboral. Al menos desde el punto de vista de estas políticas públicas, parece muy difícil distinguir entre la historia del estado del bienestar entendido como como well-being y el estado de bienestar, comprendido como welfare $^{26}$.

En términos generales, estos cambios en la comprensión de la salud, consecuencia del aumento demográfico y de la creciente industrialización, han sido estudiados de acuerdo con dos metodologías distintas: en primer lugar, por medio de la lectura de tratados y manuales relacionados con los cuidados del cuerpo, incluyendo catecismos de la salud y libros de auto-ayuda ${ }^{27}$. En segundo lugar, a través del estudio de las políticas relacionadas con medidas higiénicas o sanitarias, ya sea en contextos nacionales o coloniales ${ }^{28}$. Frente a estas dos aproximaciones, que corresponderían a una historia intelectual o social del bienestar, cabría una posibilidad diferente, capaz de combinar la historia de las formas objetivas y subjetivas de la salud. Esta última aproximación parte de la premisa de que la relación entre los elementos subjetivos y objetivos del bienestar no se mantiene constante y que, junto con las variaciones objetivas, estu-

23 BRODSKY, Phyllis L.: The Control of Childbirth: Women versus Medicine through the Ages / Phyllis L. Brodsky; Foreword by Mary Ann Shah, Jefferson (NC)-London, McFarland \& Co, 2008; MORICEAU, Caroline: Les douleurs de l'industrie: l'hygiénisme industriel en France, 1860-1914, 1 vols., En temps \& lieux 12, ParÍs: Éditions de 1'École des Hautes Études en Sciences Sociales, 2009; GRELL, Ole Peter, CUNNINGHAM, Andrew Y jutte Robert: Health Care and Poor Relief in 18th and 19th Century Northern Europe, Aldershot, Ashgate, 2002; GÉLIS, Jacques: L'Arbre et le fruit: la naissance dans l'Occident moderne, XVIe-XIXe siècle, París, Fayard, 1984; GÉLIS, Jacques: La Sage-femme ou le médecin: une nouvelle conception de la vie, París, Fayard, 1988; LAGET, Mireille: Naissances: l'accouchement avant l'âge de la clinique, París, Éditions du Seuil, 1982; GÉLIS, Jacques, LAGET, Mireille y MOREL, Marie-France (eds.): Entrer dans la vie: naissances et enfances dans la France traditionnelle, Paris, Gallimard-Julliard, 1978.

24 DUVIVIER, Franck Thenard (ed.) : Hygiène, santé et protection sociale: de la fin du XVIIIe siècle à nos jours, París, Ellipses, 2012; PORTER, Dorothy: History of Public Health and the Modern State, Amsterdam, Rodopi, 1994.

25 LABISCH, Alfons: Homo Hygienicus: Gesundheit Und Medizin in Der Neuzeit. Frankfurt \& New York, Campus Verlag, 1992.

26 BOURDELAIS, Patrice : Une peur bleue: Histoire du choléra en France (1832-1854), Paris, Payot, 1987.

27 Como en el caso del profesor Bergdolt.

28 RODRÍGUEZ OCAÑA, Esteban: Salud pública en España: ciencia, profesión y política, siglos XVIIIXX, Granada, Universidad de Granada, 2005. 
diadas hasta ahora, pueden contemplarse también cambios de naturaleza evaluativa ${ }^{29}$. Aun cuando las modulaciones culturales de la experiencia están también presentes en otros grandes problemas sociales del mundo contemporáneo, como la medida del riesgo o la seguridad, nuestra investigación se centra en la relación entre los indicadores objetivos de la salud y la percepción subjetiva del bienestar ${ }^{30}$.

\subsection{El caso de la fibromialgia}

Quizá no haya mejor campo de pruebas para comprender la tensión entre las formas históricas de objetivación de la experiencia y las cualidades subjetivas de la enfermedad que la que se expresa en torno a las consideraciones tanto públicas como privadas relacionadas con enfermedades elusivas. Dentro de la historia cultural de esa tensión no resuelta entre los indicadores objetivos de las salud y los síntomas subjetivos del malestar, la historia de la fibromialgia merece un lugar preeminente ${ }^{31}$. Se trata, en primer lugar, de una condición sin rastros visibles ni lesiones morfológicas aparentes. En este sentido, comparte rasgos definitorios con otras enfermedades similares, cuya existencia ha sido históricamente cuestionada. Tanto si hablamos de la historia de la neurastenia o de la hipocondriasis, la historia de la enfermedad no coincide, ni mucho menos, con la historia de sus síntomas ${ }^{32}$. En todos los casos, el problema reside en la ausencia de acuerdo entre lo que la medicina entiende que es "estar bien", y lo que el paciente percibe como "sentirse mal". Al mismo tiempo, al enfermedad se manifiesta

29 HERVÁS, Gonzalo y VÁZQUEZ: "Construction and Validation of a Measure of Integrative WellBeing in Seven Languages: The Pemberton Happiness Index," Health and Quality of Life Outcomes 11, no. 1 (2013), p. 66, doi:10.1186/1477-7525-11-66; DIENER, Ed, HELLIWELL, John F. y KAHNEMAN, Daniel: International Differences in Well-Being, Oxford, Oxford University Press, 2010; DIENER, Ed y SUH, Eunkook M.: Culture and Subjective Well-Being, Cambridge (MA)-Londres, MIT Press, 2000; JOSHANLOO, Mohsen: "A Comparison of Western and Islamic Conceptions of Happiness," Journal of Happiness Studies 14, no. 6 (2013), pp. 1857-1874, doi:10.1007/s10902-012-9406-7; LU, Luo y GILMOUR, Robin: "Culture and Conceptions of Happiness: Individual Oriented and Social Oriented Swb," Journal of Happiness Studies 5, no. 3 (2004), pp. 269-291, doi:10.1007/s10902-004-8789-5; BUUNK Bram y GIBBONS, Frederick X.: Health, Coping, and Well-Being: Perspectives from Social Comparison Theory, Mahwah (NJ)-Londres, Lawrence Erlbaum Associates, 1997; CHIAO, Joan Y: Cultural Neuroscience: Cultural Influences on Brain Function, Amsterdam-Londres, Elsevier, 2009.

30 BECK, Ulrich: Risk Society: Towards a New Modernity, Londres, Sage, 1992; DOUGLAS, Mary y WILDAVSKY, Aaron: Risk and Culture: An Essay on the Selection of Technical and Environmental Dangers, Berkeley-Londres, University of California Press, 1983.

31 Sobre enfermedades elusivas, HACKING, Ian: Mad Travellers: Reflections on the Reality of Transient Mental Illness, Londres, Free Association, 1999.

32 Véase BERRIOS, Germán: "Hypocondriasis: History of the Concept," en Vladan Sarcevic and Don R. Lipstitt Hypochondriasis. Modern Perspectives on an Ancient Malady, Oxford, Oxbow Books, 2001; WOLFE, Frederick et al., "The American College of Rheumatology Preliminary Diagnostic Criteria for Fibromyalgia and Measurement of Symptom Severity," Arthritis Care \& Research 62, no. 5 (2010), pp. 600-610, doi:10.1002/acr.20140; WOLFE, Frederick: "New American College of Rheumatology Criteria for Fibromyalgia: A Twenty-Year Journey," Arthritis Care \& Research 62, no. 5 (2010), pp. 583-584, doi:10.1002/ acr.20156; ESCUDERO-CARRETERO, María José et al., "Fibromialgia: percepción de pacientes sobre su enfermedad y el sistema de salud. Estudio de investigación cualitativa," Reumatología Clínica 6, no. 1 (2010), pp. 16-22, doi:10.1016/j.reuma.2009.04.008; CLAUW, Daniel J.: "Fibromyalgia: A Clinical Review," JAMA: The Journal of the American Medical Association 311, no. 15 (2014), pp. 1547-1555, doi:10.1001/ jama.2014.3266. 
a través de un espacio emocional que involucra a pacientes, médicos, cuidadores y familiares.

La contestación a los modelos biomecánicos de la salud ha visto florecer líneas de investigación preocupadas con la voz del paciente, pero también con el papel que las humanidades pueden tener en el desarrollo del conocimiento médico y de la práctica clínica. El punto de inflexión proviene tanto de la medicina narrativa como de la antropología médica. En ambos casos se acepta y comparte que el conocimiento de la enfermedad no depende tan solo del uso de instrumental o de elementos susceptibles de introducir una objetivad mecánica, sino de las formas narrativas, incluidas las historias clínicas, en las que se expresan y configuran esas mismas experiencias ${ }^{33}$. Las decisiones clínicas y las prácticas de cuidado requieren, por tanto, de un conocimiento holístico, no ajeno a aptitudes interpretativas y empáticas.

Con el fin de abrir una línea de investigación y transferencia en el marco de las humanidades médicas, el grupo HIST-EX ha propuesto una serie de actividades de cooperación con la Unidad de Fibromialgia y Fatiga Crónica del Hospital de La Ribera, en Alzira (Valencia). El objetivo de todas ellas sigue el principio general de las humanidades médicas: mejorar el bienestar de los pacientes, así como facilitar una mejor práctica clínica. Los objetivos, metodología y líneas de actuación de este proyecto entroncan con los aspectos programáticos de los retos sociales abordados desde Europa. Al tiempo que compartimos la distinción entre las ciencias de la identidad y las ciencias de la intervención, nos posicionamos claramente en este segundo programa, aspirando a ser un ejemplo de investigación orientada a la resolución de problemas de relevancia social antes que a la configuración de disciplinas. ${ }^{34}$ Dentro de esta política de cooperación intersectorial, sobre todo en el campo de las ciencias de la salud y de las humanidades médicas, planteamos la interacción con médicos y pacientes, por medio de talleres presenciales y dinámicos. En segundo lugar, proyectamos la realización y producción de documentales. Finalmente, nuestro proyecto también hace uso de innovaciones en nuevas tecnologías de la información para reducir el denominado "Treatment Gap". En todos los casos, defendemos que un grupo de investigación en humanidades pueda colaborar con un equipo clínico en la comprensión de una enfermedad, así como en el desarrollo de instrumentos terapéuticos.

33 La importancia de las Humanidades Médicas se pone de manifiesto en programas internacionalmente avalados, como es el caso de la formación en Medicina Narrativa propuesta por la Columbia University, o los programas de investigación y formación de la Universidad de Durham y del Centre for Humanities and Health en el King's College. Véase TURNER, Victor W. y BRUNER, Edward: The Anthropology of Experience, Urbana \& Chicago, University of Illinois Press, 1986; POLKINGHORNE, Donald: Narrative Knowing and the Human Sciences, SUNY Press, 1988.

34 (The future of the Social Sciences and Humanities in Europe: collected LERU papers on the SSH research agenda, Advise Paper, September 13, pp. 5-13: Essential SSH Research for the Societal Challenge 'Health, demographic change and wellbeing' (March 2013) Main authors: Philip Spinhoven, Wim van den Doel, Katrien Maes 


\section{Conclusiones}

La historia de las emociones que surge con fuerza en distinto lugares del mundo no excluye variaciones locales, donde el énfasis y la orientación se plantean en términos diferenciados. Mientras los estudios culturales de las emociones en los Estados Unidos reconocen influencias de la antropología, de la psicología o de los estudios de performatividad, la historia de las emociones del Reino Unido y de Alemania parte de nociones provenientes en su mayor parte de la historia social, como la de "economía moral" que ahora invoca el Instituto Max Planck for Human Development, en Berlín. Al mismo tiempo, en Francia se sigue hablando de historia de las sensibilidades, más en la línea de los trabajos sobre historia cultural de los sentidos. En todos los casos, la elección terminológica ya constituye un posicionamiento teórico. En el ámbito de los estudios de emociones en España y en América Latina aflora una dimensión política que delimita tanto el objeto como la orientación del estudio. La historia del tiempo presente, la geografía urbana, la relación entre la historia y la memoria o la historia cultural del bienestar son solo algunas de las líneas de investigación de las que se nutre la historia cultural de las emociones escrita y publicada en lengua española. En el caso concreto de nuestro grupo de investigación, el estudio de las cualidades subjetivas de la experiencia nos ha hecho tomar partido por una historia de las formas culturales de la experiencia subjetiva. Este estudio de los elementos dramáticos, retóricos y políticos de la experiencia no solo se aplica a las pasiones de la modernidad, sino a conceptos tan elusivos como el de "bienestar" y a enfermedades tan discutidas como la fibromialgia. La cooperación de HIST-EX con el equipo médico de la Unidad de Fibromialgia del Hospital de La Ribera en Valencia constituye, a nuestro juicio, un indicador de la manera en que las humanidades médicas en particular, así como las Nuevas Humanidades en general, pueden intervenir en los retos sociales del siglo XXI. Si la historia de las emociones fuera una moda, como sostienen algunos académicos, habría que concluir que se trata de una moda necesaria que, al tiempo que saca a la luz la historia olvidada del pasado, abre nuevas perspectivas a las humanidades del presente.

\section{Bibliografía}

BECK, Ulrich: Risk Society: Towards a New Modernity, Londres, Sage, 1992.

BENDELOW, Gillian y WILLIAMS, Simon J.: Emotions in Social Life: Critical Themes and Contemporary Issues. Abingdon (Oxon)-Nueva York, Routledge, 2002.

BERGDOLT, Klaus. Wellbeing: A Cultural History of Healthy Living. CambridgeMalden (MA), Polity, 2008.

BERRIOS, Germán. "Hypocondriasis: History of the Concept", SARCEVIC, Vladan y LIPSTITT, Don R. (ed.): Hypochondriasis. Modern Perspectives on an Ancient Malady, Oxford, Oxbow Books, 2001. 
BOURDELAIS, Patrice : Une peur bleue: histoire du choléra en France (18321854), París, Payot, 1987.

BRODSKY, Phyllis L.: The Control of Childbirth: Women versus Medicine through the Ages, Jefferson (NC)-Londres, McFarland \& Co, 2008.

BUUNK Bram y GIBBONS, Frederick X.: Health, Coping, and Well-Being: Perspectives from Social Comparison Theory, Mahwah (NJ)-Londres, Lawrence Erlbaum Associates, 1997.

BURKE, Peter: "Is There a Cultural History of the Emotions?," en GOUK, Penelope y HILLS, Helen (eds.): Representing Emotions: New Connections in the Histories of Art, Music and Medicine, Aldershot, Ashgate, 2005, pp. 35-48.

CHIAO, Joan Y.: Cultural Neuroscience : Cultural Influences on Brain Function, Amsterdam-Londres, Elsevier, 2009.

CLAUW, Daniel J.: "Fibromyalgia: A Clinical Review", JAMA: The Journal of the American Medical Association, 311, no. 15 (2014), pp. 1547-55. doi:10.1001/ jama.2014.3266.

Cuerpos, Emociones y Sociedad. "Presentación." Revista Latinoamericana de Estudios sobre cuerpos, emociones y sociedad 1, no. 1 (2009), pp. 4-5.

DEWEY, John: Art as Experience, Londres, George Allen \& Unwin, 1934.

DÍAZ-VERA, Javier: "Emotions in the Household: Emotion Words and Metaphors in Domesday Book Personal Names," Names - A Journal of Onomastics, 2015.

_ . "On Saying Two Things at Once: The Historical Semantics and Pragmatics of Old English Emotion Words." Folia Linguistica Historica 35, no. 1 (2014).

DÍAZ-VERA, Javier y CABALLERO, Rosario: "Exploring the Feeling-Emotions Continuum across Cultures: Jealousy in English and Spanish," Intercultural Pragmatics, 10, no. 2 (2013), pp. 265-94, doi:10.1515/ip-2013-0012.

DOUGLAS, Mary: Risk and Culture: An Essay on the Selection of Technical and Environmental Dangers, Berkeley-London, University of California Press 1983.

DUVIVIER, Franck Thenard (ed.) : Hygiène, santé et protection sociale: de la fin du XVIIIe siècle à nos jours, París, Ellipses, 2012

DIENER, Ed y SUH, Eunkook M.: Culture and Subjective Well-Being, Cambridge (MA)-London, MIT Press, 2000.

DIENER, Ed, HELLIWELL, John F. y KAHNEMAN, Daniel: International Differences in Well-Being, Oxford, Oxford University Press, 2010.

ESCUDERO-CARRETERO, María José et al., "Fibromialgia: percepción de pacientes sobre su enfermedad y el sistema de salud. Estudio de investigación cualitativa," Reumatología Clínica 6, no. 1 (2010), pp. 16-22, doi:10.1016/j.reuma.2009.04.008.

FANTINI, Bernardino, MARTÍN MORUNO, Dolores y MOSCOSO, Javier: On Resentment: Past and Present, Cambridge, Cambridge Scholars, 2013.

FEBVRE, Lucien: "La sensibilité et l'histoire. Comment reconstituer la vie affective d'autrefois?," Annales d'Histoire sociale, 2 (1941), pp. 5-20. 
FREVERT, Ute et al.: Emotional Lexicons: Continuity and Change in the Vocabulary of Feeling 1700-2000, Oxford, Oxford University Press, 2014.

GÉLIS, Jacques. L'Arbre et le fruit: la naissance dans l'Occident moderne, XVIeXIXe siècle, París, Fayard, 1984.

_. La Sage-femme ou le médecin: une nouvelle conception de la vie. Paris: Fayard, 1988.

GÉLIS, Jacques, LAGET, Mireille et MOREL, Marie-France (eds.): Entrer dans la vie: naissances et enfances dans la France traditionnelle, París, GallimardJulliard, 1978.

GOLDIE, Peter: The Mess Inside: Narrative, Emotion and the Mind, Oxford, Oxford University Press, 2012.

GOUBERT, Jean-Pierre. Une histoire de l'hygiène: eau et salubrité dans la France contemporaine, Paris, Pluriel, 2011.

GRELL, Ole Peter, CUNNINGHAM, Andrew y JUTTE, Robert: Health Care and Poor Relief in 18th and 19th Century Northern Europe, Aldershot, Ashgate, 2002.

HACKING, Ian: Mad Travellers: Reflections on the Reality of Transient Mental Illness, London: Free Association, 1999.

HERVÁS, Gonzalo y VÁZQUEZ, Carmelo: "Construction and Validation of a Measure of Integrative Well-Being in Seven Languages: The Pemberton Happiness Index." Health and Quality of Life Outcomes 11, no. 1 (2013), p- 66. doi:10.1186/1477-7525-11-66.

JOSHANLOO, Mohsen: "A Comparison of Western and Islamic Conceptions of Happiness", Journal of Happiness Studies 14, no. 6 (2013), pp. 1857-1874. doi:10.1007/s10902-012-9406-7.

KOSELLECK, Reinhart: "Cambio de experiencia y cambio de método", en Los estratos del tiempo: estudios sobre la historia, Barcelona, Editorial Paidós, 2001, pp. 43-93.

LABANYI, Jo: "Doing Things: Emotions, Affect, and Materiality." Journal of Spanish Cultural Studies, 11 (2010), pp. 223-233.

LABISCH, Alfons: Homo Hygienicus: Gesundheit Und Medizin in Der Neuzeit, Frankfurt \& New York, Campus Verlag, 1992.

LAGET, Mireille. Naissances: l'accouchement avant l'âge de la clinique. L'Univers historique. Paris: Éditions du Seuil, 1982.

LU, Luo y GILMOUR, Robin: "Culture and Conceptions of Happiness: Individual Oriented and Social Oriented Swb." Journal of Happiness Studies 5, no. 3 (2004), pp. 269-91. doi:10.1007/s10902-004-8789-5.

TAUSIET, Carles Maria y AMELANG, James S. (eds.): Accidentes del alma: las emociones en la Edad Moderna, Madrid, Abada, 2009.

MARROQUÍN, Lucía Díaz. “'La nieve arder'. La retórica afectiva en el universo petrarquista de la Zarzuela Acis y Galatea", Revista de Literatura 66, no. 132 (2004), pp. 431-64. 
La retórica de los afectos. Edition Reichenberger, 2008.

MATT, Susan J. y STEARNS, Peter N.: Doing Emotions History, Urbana, University of Illinois Press, 2013.

MEDINA DOMÉNECH, Rosa María: Ciencia y sabiduría del amor : una historia cultural del franquismo (1940-1960), Madrid-Frankfurt am Main, Iberoamericana, 2013.

MORICEAU, Caroline : Les douleurs de l'industrie: l'hygiénisme industriel en France, 1860-1914, París, Éditions de l’École des hautes études en sciences sociales, 2009.

MOSCOSO, Javier: "Poétique, rhétorique et politique des émotions : le drame de l'expérience", en LANGUE, Frédérique y CAPDEVILLE, Luc : Le passé des émotions. D'une histoire à vif en Espagne et Amérique Latine, editado por Frédérique Langue and Luc Capdeville. Rennes: Presses Universitaires de Rennes, in press.

NUSSBAUM, Martha C.: Political Emotions: Why Love Matters for Justice. Cambridge, The Belknap Press of Harvard University Press, 2013.

PINCHEIRA, Pincheira (ed.): Archivos de frontera. El Gobierno de las emociones en Argentina y Chile del Presente. Santiago de Chile, Ediciones Escaparate, 2012.

PINHEIRO KOURY, Mauro Guilherme. "Surge Uma Revista Em Sociologia Das Emoções." Revista Brasileira de Sociologia Da Emoçao 1, no. 1 (2002): 4-5.

POLKINGHORNE, Donald. Narrative Knowing and the Human Sciences, SUNY Press, 1988.

PORTER, Dorothy. History of Public Health and the Modern State. Amsterdam: Rodopi, 1994.

RODRÍGUEZ OCAÑA, Esteban: Salud pública en España: ciencia, profesión y politica, siglos XVIII-XX, Granada, Universidad de Granada, 2005.

ROSENWEIN, Barbara H. Emotional Communities in the Early Middle Ages, Ithaca \& London: Cornell University Press, 2006.

- "Problems and Methods in the History of Emotions." Passions in Context 1 (2010): $1-32$.

. "Worryng about Emotions in History." The American Historical Review 107 (2002): $821-845$.

SABIDO RAMOS, Olga. "El cuerpo y la afectividad como objetos de estudio en América Latina: intereses temáticos y proceso de institucionalización reciente" Sociológica (México) 26, no. 74 (2011), pp. 33-78.

SCHEER, Monique. "Are Emotions a Kind of Practice (and Is That What Makes Them Have a History)? A Bourdieuan Approach to Defining Emotion", History and Theory, 51 (2012), pp. 193-220.

SIECE, Publicado por. "GRAFOSFERA: Conocer, Conmover.” Accessed May 30, 2014. http://grafosfera.blogspot.co.uk/2010/03/conocer-conmover.html.

TURNER, Victor W., and Edward. Bruner. The Anthropology of Experience. Urbana \& Chicago, University of Illinois Press, 1986. 
WOLFE, Frederick. "New American College of Rheumatology Criteria for Fibromyalgia: A Twenty-Year Journey." Arthritis Care \& Research 62, no. 5 (2010), pp. 583-584. doi:10.1002/acr.20156.

WOLFE, Frederick et al. "The American College of Rheumatology Preliminary Diagnostic Criteria for Fibromyalgia and Measurement of Symptom Severity", Arthritis Care \& Research 62, no. 5 (2010), pp. 600-610. doi:10.1002/acr.20140.

ZARAGOZA BERNAL, Juan Manuel: "Historia de las emociones: una corriente historiográfica en expansión”, Asclepio 65, no. 1 (2013): e012. doi:10.3989/asclepio.2013.12. 